\title{
DESIGN PARA A SUSTENTABILIDADE E O CICLO DE VIDA DOS MATERIAIS: UMA REFLEXÃO ACERCA DA PRODUÇÃO DE COMPÓSITOS BIODEGRADÁVEIS
}

\author{
Eliana Paula Calegari \\ Universidade Federal do Rio Grande do Sul \\ elianapaulac@gmail.com \\ Branca Freitas de Oliveira \\ Universidade Federal do Rio Grande do Sul \\ branca@ufrgs.br
}

\begin{abstract}
Resumo
Existem materiais que podem causar menos danos ambientais, como os biodegradáveis, por possuírem a vantagem de degradarem-se em um curto período de tempo. Sendo assim, o objetivo do presente trabalho é discutir o ciclo de vida dos compósitos biodegradáveis e sua relação com o impacto ambiental na produção destes materiais. A partir de pesquisa bibliográfica, constatou-se que no desenvolvimento dos compósitos biodegradáveis são utilizados materiais de fontes renováveis, aditivos e tratamentos que visam conservar a propriedade de biodegradação, além disso, são empregados métodos convencionais de processamento que devido aos materiais naturais utilizados, diminuem os danos nos equipamentos. Dessa forma, o ciclo de vida dos compósitos biodegradáveis direciona-se para o baixo impacto ambiental.
\end{abstract}

Palavras-chave: sustentabilidade, ciclo de vida, compósitos biodegradáveis, biodegradação, impacto ambiental.

\footnotetext{
Abstract

There are materials that cause less environmental damage, such as biodegradable, so they have the advantage that they degrade in a short period of time. Thus, the aim of this paper is to discuss the life cycle of biodegradable composites and its relation to the environmental impact in the production of these materials. From literature, it was found that in the development of biocomposites materials from renewable sources, additives and treatments aimed at preserving the property of biodegradation are used, in addition, conventional methods are employed to process due to the
} 
natural materials decrease in equipment damage. Thus, the life cycle of biocomposites is directed to the low environmental impact.

Keywords: Sustainability, life cycle, biocomposites, biodegradation, environmental impact.

\section{INTRODUÇÃO}

Com o avanço da ciência e da tecnologia, surgiram e continuam surgindo inúmeros novos materiais. Existem aproximadamente 100 mil materiais, essa gama permite que o design seja inovador a partir da exploração imaginativa dos novos e aprimorados materiais. Este número tende a aumentar principalmente pelo fato de que as pessoas estão mais conscientes das questões relacionadas à seleção de materiais em razão do surgimento do conceito de sustentabilidade (ASHBY e JOHNSON, 2010).

Manzini e Vezzoli (2002) caracterizam a dimensão ambiental da sustentabilidade segundo condições, as quais, em níveis regionais e planetários, as atividades humanas não devem interferir nos ciclos naturais. Nas palavras de Kazazian (2005), "vivemos uma 'idade' em que se impõe o desenvolvimento sustentável, estando ao nosso alcance imaginar soluções que favoreçam a prosperidade sem que isso implique regredir em qualquer setor dos sistemas econômico ou natural" (p.7). Assim, a preocupação com o meio ambiente, sucedida da degradação ambiental e a falta de recursos naturais, exige novas soluções imprescindivelmente sustentáveis.

De acordo com Beylerian e Dent (2007), há inúmeras pessoas que trabalham na próxima geração de materiais, processos e ferramentas que permitirão o desenvolvimento de novos projetos voltados para a preservação ambiental. Para os autores, essa inovação em materiais é visivelmente necessária para resolver os problemas e as necessidades do futuro, principalmente com a preocupação das questões ecológicas e com a escassez de materiais provenientes de fontes não renováveis. Cada vez mais a abordagem no desenvolvimento de novos materiais compreende todo o ciclo de vida dos produtos, em que considera os impactos causados desde a matéria-prima até o destino final do produto (CARVALHO, 2011).

As preocupações com as questões ecológicas têm gerado interesse na utilização de fontes renováveis, sustentáveis e de materiais que podem ser compostados. Desta maneira, o descarte destes materiais torna-se relevante para a minimização de impactos ambientais, pois ao final de seu ciclo de vida podem ser colocados em aterros sanitários (RAMÍREZ, 2011).

Nos dias atuais estão sendo desenvolvidos materiais que podem ser compostados com segurança no final de sua vida útil, como é o caso dos compósitos biodegradáveis, que normalmente são preparados a partir de fontes renováveis, o que permite a biodegradação após o descarte (MOHANTY et al., 2005). No entanto, apesar destes materiais serem biodegradáveis, que é uma propriedade que vai ao encontro da sustentabilidade, é preciso analisar todo o ciclo de vida deste material, ou seja, do seu processamento até o descarte.

Desta forma, o objetivo do presente trabalho é discutir o ciclo de vida dos compósitos biodegradáveis e sua relação com o impacto ambiental na produção destes 
materiais. Assim, a partir de pesquisa bibliográfica, em publicações sobre o desenvolvimento de compósitos biodegradáveis, foram levantados dados referentes aos materiais e o processamento destes materiais, o que permitiu a análise do ciclo de vida, principalmente em relação à etapa de produção e o descarte.

\section{DESENVOLVIMENTO}

Para analisar o ciclo de vida dos compósitos biodegradáveis foram utilizadas as etapas propostas por Manzini e Vezzoli (2002): pré-produção, produção, distribuição, uso e descarte de produtos. Nos entanto, os compósitos biodegradáveis são materiais recentes que se encontram em fase de desenvolvimento e caracterização, e não foram encontradas aplicações na indústria. Dessa forma, a análise do ciclo de vida destes materiais concentra-se, principalmente, na produção, que envolve os materiais utilizados, a preparação a partir dos tipos de tratamentos utilizados nas fibras, os aditivos empregados na matriz e o tipo de processamento.

Os compósitos biodegradáveis podem ser constituídos por diferentes tipos de materiais das fibras e da matriz bem como, podem ser processados de diferentes maneiras. Desse modo, foram coletados 50 artigos que objetivam o desenvolvimento de compósitos biodegradáveis. Devido ao grande volume de publicações foi necessário delimitar a busca, em que foi focada em pesquisas que desenvolvem estes materiais a partir das seguintes fibras vegetais brasileiras: carnaúba, cana de açúcar, juta, banana, curauá, sisal, casca de arroz, palha de milho e de trigo, e fibra de coco.

A partir disso, foram coletados os artigos principalmente nos seguintes periódicos: Material and Design, Carbohydrate polymers, Composites Science and Tecnology, Industrial Crops and Products, Biomass and Bioenegy, Process Biochemistry, Journal de Composites Material, Journal of Polymers and Environment, Composites, Polymer Composites, Polímeros, Journal of Reinforced Plastics \& Composites, Bioresource Technology. As palavras-chave utilizadas para a busca dos artigos foram baseadas na delimitação por meio das fibras vegetais, assim, partiu-se das palavras biodegradable composite e biocomposite, encontradas com frequência nos artigos e tidas como sinônimas, cruzando-as com o nome das fibras, conforme ilustra a figura 1.

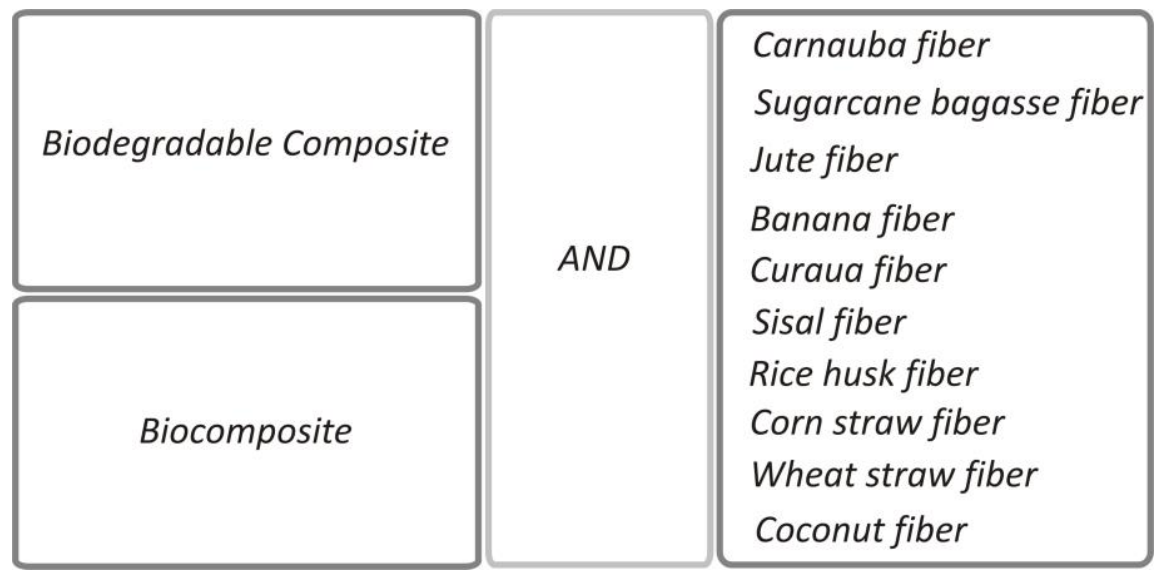

Figura 1 - Palavras-chave utilizadas na pesquisa dos artigos.

Fonte: Elaborado pelas autoras, com base na pesquisa realizada.

Dessa maneira, foi possível verificar os tipos de materiais, os tratamentos utilizados nas fibras, os aditivos empregados na matriz e o tipo de processamento dos compósitos biodegradáveis. A partir destes dados, foi possível analisar, principalmente, 
a fase de produção do ciclo de vida destes materiais e o descarte.

\subsection{Os materiais utilizados para a produção de compósitos biodegradáveis}

Existe uma grande variedade de tipos de materiais que constituem as fibras e matrizes utilizadas para a produção dos compósitos biodegradáveis, no entanto, como já mencionado, delimitou-se a busca de artigos que visam à pesquisa por compósitos biodegradáveis constituídos por fibras brasileiras.

Na maioria dos estudos há variação na porcentagem de fibras utilizadas, tipos de tratamentos na fibra e aditivos na matriz e diferentes formas de processamento. Isso ocorre pelo fato de que os artigos propõem-se a verificação das quantidades de fibras e métodos de processamento que conferem melhores propriedades para os compósitos biodegradáveis, bem como, a diminuição da degradação e absorção de água.

\subsubsection{Tipos de matrizes}

As matrizes utilizadas para o desenvolvimento de materiais biodegradáveis podem ser produtos da biomassa, a partir de micro-organismos, da biotecnologia e de produtos petroquímicos que na maioria dos casos são processados e transformados em biopolímeros (AVÉROUS; BOQUILLON, 2004). Nos estudos mapeados, a maior parte das matrizes utilizadas para o desenvolvimento de compósitos biodegradáveis são materiais de fontes renováveis oriundas de produtos da biomassa, como pode ser observado na figura 2 , que apresenta os tipos de matrizes utilizadas no desenvolvimento de compósitos biodegradáveis dos estudos levantados.

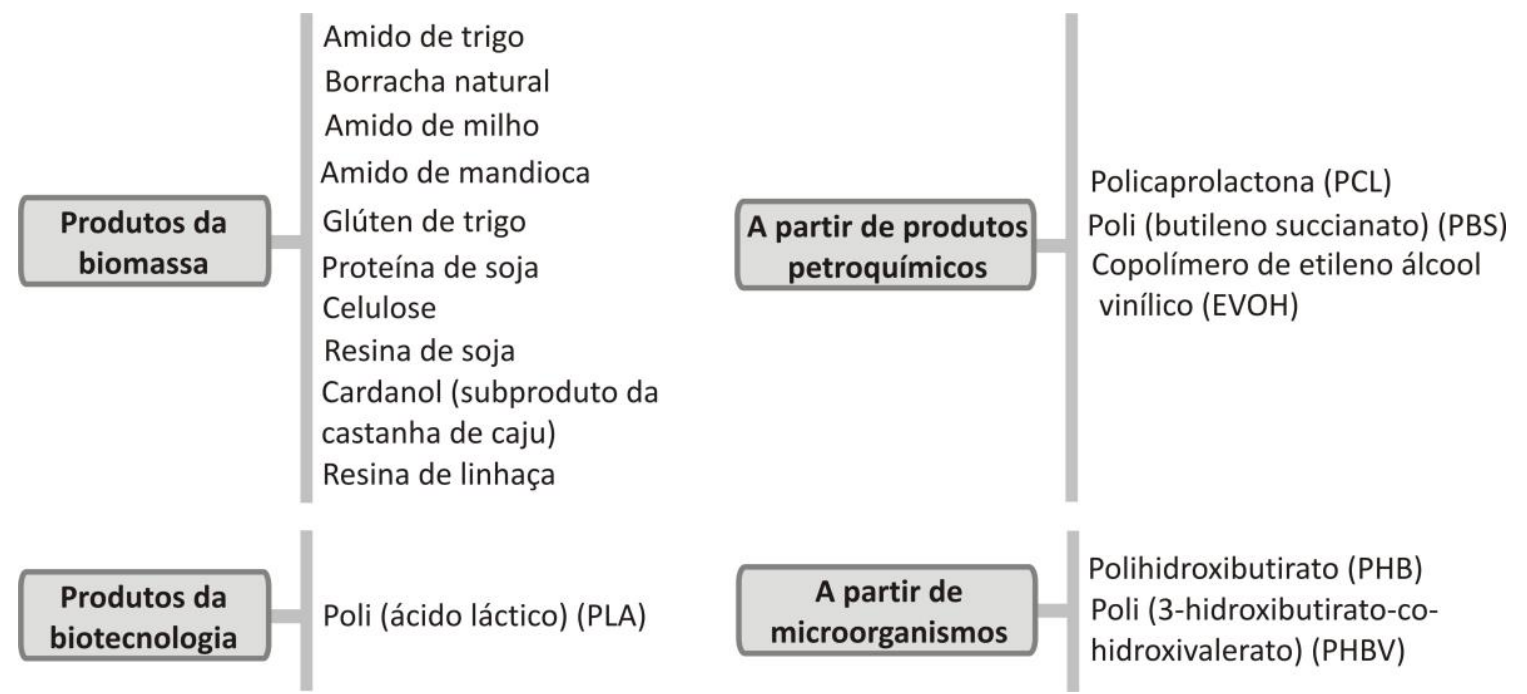

Figura 2 - Matrizes utilizadas no desenvolvimento de compósitos biodegradáveis nos estudos mapeados.

Fonte: Elaborado pelas autoras, com base na pesquisa realizada.

A constatação da prevalência da utilização de produtos da biomassa para compor as matrizes de compósitos biodegradáveis pode ocorrer pelo fato de que os polímeros naturais mais pesquisados originam-se do amido (AVÉROUS; DIGABEL, 2006). Em consonância, para Rosa et al. (2009), o amido é um recurso agrícola largamente estudado e promissor para a produção de biopolímeros como matrizes para 
empregar em compósitos biodegradáveis.

\subsubsection{Tipos de fibras}

As fibras vegetais possuem potencial para serem empregadas em compósitos biodegradáveis, pois possuem boa estabilidade térmica e boas propriedades mecânicas (MOHANTY et al., 2000). O levantamento de artigos que desenvolvem e caracterizam compósitos biodegradáveis partiu de estudos que tratam sobre fibras vegetais brasileiras. Desse modo, as pesquisas abordam diferentes tipos de fibras de várias partes das plantas, como pode ser visualizado na figura 3.

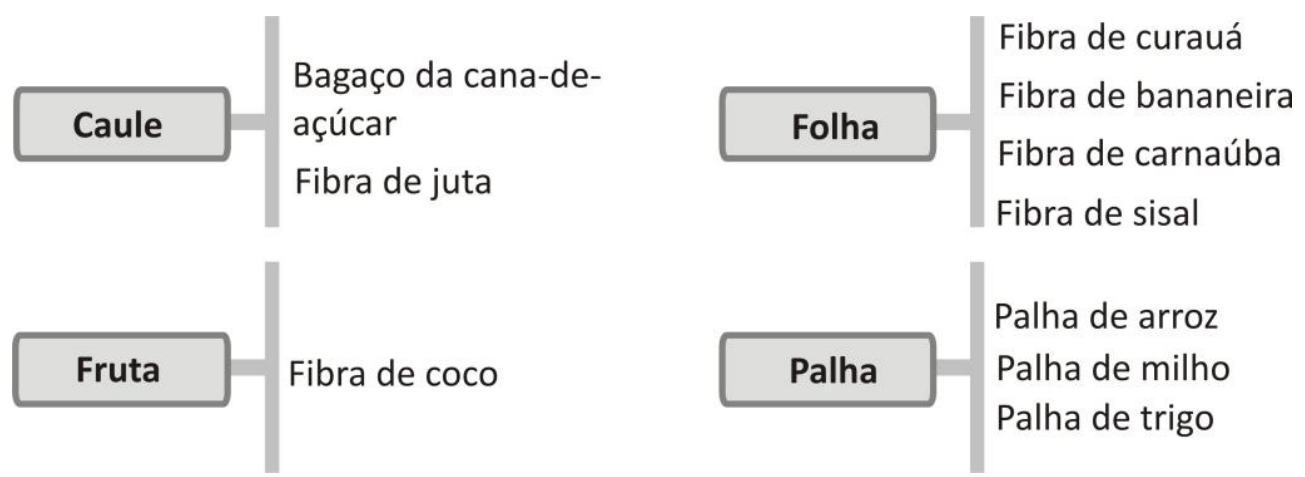

Figura 3 - Fibras vegetais utilizadas no desenvolvimento de compósitos biodegradáveis nos estudos mapeados.

Fonte: Elaborado pelas autoras, com base na pesquisa realizada.

A maior parte dos estudos levantados estão concentrados no desenvolvimento de compósitos com fibras de coco. Acredita-se que haja bastante interesse em pesquisas relacionadas com esse tipo de fibra pelo fato de que a fibra é um subproduto do coco, ou seja, é um resíduo, sendo este o principal motivo utilizado pelos pesquisadores para justificar o emprego deste tipo de fibra como reforço para os compósitos. As fibras provenientes do bagaço da cana-de-açúcar, de palha de arroz, milho e trigo também são consideradas resíduos, e na agroindústria são muitas vezes tratadas como entulho. Isso mostra a relevância das pesquisas que buscam um destino para esse material.

\subsection{A preparação dos compósitos biodegradáveis}

De acordo com Satyanarayana (2010), os fatores necessários para a produção de compósitos biodegradáveis incluem a seleção das fibras vegetais e da matriz, tratamentos de superfície adequados nas fibras, para uma maior aderência da fibra e da matriz e a redução da sensibilidade à umidade, e as técnicas de fabricação devem ser preferencialmente de baixo custo.

Nos artigos mapeados constatou-se que o desenvolvimento de compósitos biodegradáveis envolve inicialmente os tratamentos nas fibras para que estas adquiram melhores propriedades bem como diminuam a absorção de água. Além disso, geralmente são adicionados aditivos na matriz também com o intuito de melhorar as propriedades. Após este processo inicial, de preparação dos materiais da matriz e do reforço, eles são misturados e conformados, como será descrito na sequência. 


\subsubsection{Processamento}

O processamento dos materiais está completamente interligado com as propriedades (CALLISTER, 2002). Assim, os estudos sobre os compósitos biodegradáveis apontam que diferentes processamentos resultam em distintas propriedades, as quais influenciam no desempenho dos materiais. O mapeamento demonstrou que os processos de produção dos compósitos biodegradáveis são feitos pelos métodos visualizados na figura 4.

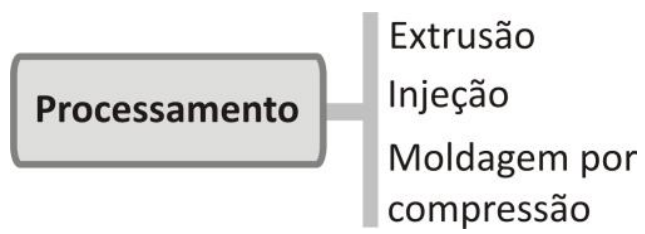

Figura 4 - Processamentos utilizados nos compósitos biodegradáveis dos estudos mapeados. Fonte: Elaborado pelas autoras, com base na pesquisa realizada.

Os compósitos biodegradáveis são fabricados a partir de tecnologia tradicional, como a extrusão, injeção e moldagem por compressão. Faruk et al. (2012) ressaltam que o processamento destes materiais inclui a extrusão, moldagem por compressão e injeção, o que vai ao encontro dos tipos de processamentos utilizados nos artigos mapeados. Contudo, os autores reportam para o fato de que tecnologias inovadoras e soluções de processos precisam ser pesquisadas para a obtenção de melhores propriedades.

\subsubsection{Tratamentos nas fibras}

Segundo Silva (2010), o interesse em utilizar fibras naturais como componentes de reforço em compósitos ocorre pelo fato de serem consideradas baratas, menos abrasivas e biodegradáveis, quando comparadas com fibras inorgânicas. Entretanto a natureza hidrofílica das fibras naturais afeta a aderência entre o reforço e a matriz pela fraca adesão interfacial. Dessa forma, a modificação da superfície das fibras por métodos físicos ou químicos pode amenizar esse problema e melhorar as propriedades dos compósitos, pois, as propriedades das fibras irão influenciar diretamente no desempenho dos compósitos biodegradáveis (FARUK et al., 2010).

Neste sentido, o principal objetivo dos tratamentos de superfície nas fibras vegetais é maximizar a força de ligação de modo a melhorar a adesão entre as fibras e a matriz (KABIR et al., 2012). Esses tratamentos geralmente são baseados na utilização de grupos funcionais reagentes que são capazes de reagir com a estrutura das fibras e mudar sua composição, ou seja, os tratamentos químicos expõem grupos funcionais reativos sobre a superfície das fibras e, assim, favorece o acoplamento com a matriz. Como resultado, a absorção de umidade das fibras pode ser reduzida, o que facilita a compatibilidade com a matriz (ABDELMOULEH et al., 2007). Portanto, tratamentos sobre a superfície das fibras naturais são necessários para melhorar as propriedades dos compósitos biodegradáveis, assim, os estudos mapeados utilizam os tratamentos apresentados na figura 5 . 


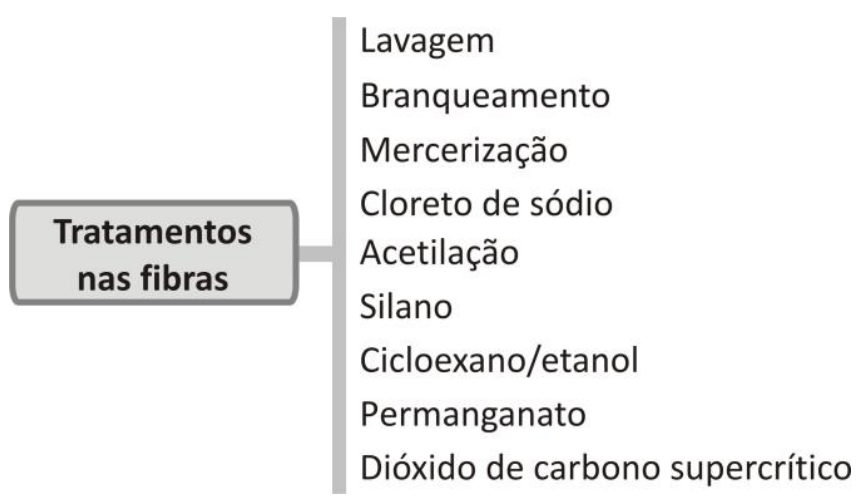

Figura 5 - Tipos de tratamentos utilizados nas fibras vegetais dos estudos mapeados. Fonte: Elaborado pelas autoras, com base na pesquisa realizada.

Dentre os tratamentos utilizados nos estudos, percebeu-se que os mais empregados são: a lavagem, a mercerização e o braqueamento. A lavagem consiste em um banho com água nas fibras e contribui para a eliminação de resíduos indesejáveis na superfície ativando-a para a sua utilização em compósitos. A mercerização baseia-se no tratamento das fibras com hidróxido de sódio $(\mathrm{NaOH})$ e o branqueamento geralmente é feito com solução de peróxido alcalino, sendo que estes tratamentos são amplamente utilizados para modificar a estrutura molecular da celulose (CAMPOS et al., 2011).

$\mathrm{Na}$ maioria dos estudos, as fibras tratadas tiveram melhoria na resistência mecânica e estabilidade dimensional dos compósitos resultantes em comparação com as puras. Geralmente, diferentes tratamentos na superfície das fibras são realizados em conjunto, como ocorreu na pesquisa realizada por Campos et al. (2011), em que foi utilizada a lavagem com água e posteriormente o branqueamento.

\subsubsection{Aditivos na matriz}

Além dos tratamentos na superfície das fibras vegetais, são adicionados aditivos na matriz dos compósitos biodegradáveis para melhorar as suas propriedades. Os principais aditivos utilizados são os plastificantes, que consistem em substâncias empregadas durante o processamento de materiais poliméricos que visam melhorar a flexibilidade e em geral a processabilidade. Basicamente, são solventes não voláteis com massa molecular menor do que o próprio polímero, que quando adicionados ao polímero o tornam mais macio e flexível formando um material mais homogêneo (SCHLEMMER, 2007). A figura 6 ilustra os aditivos utilizados nas pesquisas mapeadas dos compósitos biodegradáveis.

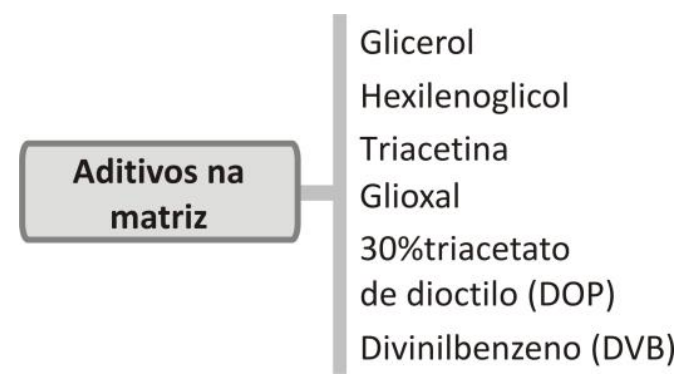

Figura 6 - Tipos de aditivos utilizados na matriz dos compósitos biodegradáveis dos estudos mapeados.

Fonte: Elaborado pelas autoras, com base na pesquisa realizada. 
A partir do mapeamento percebeu-se que na maioria das pesquisas é utilizado o glicerol como plastificante na composição dos compósitos biodegradáveis. Os projetos que visam à produção de polímeros de origem vegetal e que utilizam a química verde e renovável empregam o glicerol nos biopolímeros, a glicerina é o nome comercial do glicerol, que pode ser produzido a partir de óleos vegetais e como subproduto do biodiesel (OLIVEIRA, 2013).

\subsection{Ciclo de vida dos compósitos biodegradáveis}

A partir do levantamento dos materiais e o processamento utilizados no desenvolvimento de compósitos biodegradáveis foi possível verificar o ciclo de vida destes materiais, principalmente nas etapas de produção e descarte. Em relação aos materiais utilizados nas matrizes dos compósitos, grande parte são provenientes da biomassa e são recursos renováveis, no entanto, a maioria são utilizados para a alimentação, como o milho, a batata, a mandioca e outros. Desse modo, existem preocupações em relação à demanda de materiais verdes em relação à distorção do mercado de alimentos, ou seja, quando é utilizado solo para produção de matériaprima para o desenvolvimento de materiais, provavelmente o espaço para o plantio de alimentos poderá diminuir. Segundo Vilaplana et al. (2010), destaca-se, que a matériaprima da biomassa que se destina a tais processos deve ser selecionada de modo que não interfira nos custos de fornecimento de alimentos.

Para que os materiais utilizados na matriz dos compósitos biodegradáveis tenham melhores desempenhos, são adicionados aditivos. Na maioria dos estudos que visam à preparação dos compósitos foi utilizado o glicerol, que é considerado um plastificante renovável, que pode substituir aditivos utilizados pela indústria que são tóxicos e visam conferir maciez e flexibilidade aos produtos feitos principalmente de policloreto de vinila (PVC). Segundo a reportagem de Oliveira (2013), a professora Sônia Faria Zawadzki, do Departamento de Química da Universidade Federal do Paraná (UFPR), afirma que o glicerol é um plastificante renovável com base em óleo vegetal que pode substituir os ftalatos que são produzidos a partir de derivados de petróleo. Isso é de extrema importância, pois os ftalatos são substâncias que estão associados ao câncer, má-formação esquelética, problemas endócrinos e hormonais.

Em se tratando dos materiais utilizados nas fibras, estes são oriundos de fontes naturais, baratas, menos abrasivas e biodegradáveis, geralmente, provenientes de resíduos da agroindústria, como a palha de arroz, de milho e de trigo, a cana-de açúcar e outros. Sendo assim, a utilização deste tipo de material para a produção de compósitos biodegradáveis pode ser positivo já que se trata do aproveitamento de resíduos, além de serem provenientes de fontes renováveis. Em relação aos tratamentos utilizados nas fibras, que visam melhorar as propriedades dos compósitos biodegradáveis, na maioria dos estudos foi utilizada a lavagem com água, o que não resulta em poluição por resíduos químicos.

Os compósitos briodegradáveis dos artigos mapeados são processados por métodos comumente utilizados na indústria, como: extrusão, injeção e moldagem por compressão. Segundo Mohanty et al. (2005), os compósitos biodegradáveis podem ser injetados, sendo que estes materiais são menos prejudiciais aos equipamentos de fabricação. Em consonância, Satyanarayana (2010) destaca que o processamento de compósitos biodegradáveis é atrativo por ser menos abrasivo, o que pode diminuir o risco de danificação de equipamentos, em comparação com compósitos de fibra de 
vidro, além da ausência de partículas suspensas no ar, reduzindo problemas respiratórios nos trabalhadores.

Outro ponto importante do ciclo de vida dos compósitos biodegradáveis é o descarte. De acordo com Plackett et al. (2003), o desenvolvimento de compósitos biodegradáveis parte de exigências de materiais que podem ser reciclados e/ou que podem biodegradarem-se em segurança no meio ambiente, no final da sua vida útil. Dessa forma, a melhor forma de descarte dos produtos fabricados com este material é a compostagem já que ele é biodegradável, no entanto, é importante destacar que durante o processo de fabricação dos produtos deve-se atentar para o fato de usar outros materiais sintéticos e até mesmo tipos de acabamentos, pinturas que não sejam biodegradáveis. Isso se deve ao fato de que o compósito biodegradável pode perder sua propriedade de biodegradação, ocasionando maior poluição e degradação no meio ambiente.

Cabe ressaltar que a utilização de materiais renováveis para a fabricação de compósitos biodegradáveis não garante a isenção de impactos ambientais. É preciso levar em consideração os procedimentos de processamento empregados em conjunto com a evidência de que não há efeitos toxicológicos durante sua vida útil bem como os procedimentos na gestão de resíduos no fim da vida do produto. Desta forma, para verificar o nível de impacto dos compósitos biodegradáveis é preciso considerar todo o ciclo de vida do produto, ou seja, a partir da origem da matéria-prima até a gestão dos resíduos, para garantir a sua melhoria frente ao meio ambiente e como alternativa aos materiais tradicionais.

Neste contexto, há considerações sociais e econômicas importantes na seleção dos meios e processos para a fabricação de compósitos biodegradáveis. Desta maneira, é primordial a seleção cuidadosa da terra usada para a produção de biomassa, em termos de seu impacto sobre a disponibilidade de recursos naturais, entre eles, o solo, a matéria orgânica, os nutrientes e a água, o destino dos elementos químicos associados através dos ciclos ambientais, o impacto sobre o clima, e o efeito sobre os animais e os seres humanos.

\section{CONCLUSÃO}

A análise do ciclo de vida dos compósitos biodegradáveis, focada na etapa de produção, revelou que estes materiais estão sendo desenvolvidos de maneira a diminuir os impactos ambientais, pois constatou-se que são empregados recursos de fontes renováveis, a química verde, e a matéria-prima geralmente proveniente de resíduos da agroindústria e de origem local. Destaca-se que a produção destes materiais influencia o seu descarte, ou seja, na medida em que os compósitos biodegrádaveis são produzidos com materiais e com métodos que visam à diminuição de impactos ambientais a sua propriedade de biodegadabilidade não é afetada, assim, o descarte destes materiais podem ser feitos por meio da compostagem, o que ocasiona a diminuição de lixo em aterros sanitários.

No entanto, estes materiais encontram-se em nível de pesquisa, e com o desenvolvimento em maiores escalas, é importante manter estas características para que continuem sendo desenvolvidos sob esta ótica. Com isso, as biorrefinarias futuras, com base em operações na química verde podem ser a base para o fornecimento de materiais que visam menos impactos para a produção de compósitos biodegradáveis. Assim, estes materiais podem ser desenvolvidos de acordo com a perspectiva da 
sustentabilidade na medida em que cumprem os requisitos de desenvolvimento para uma produção verde e levem em conta todo o ciclo de vida do produto.

\section{REFERÊNCIAS}

ABDELMOULEH, M.; BOUFIS, S.; BELGACEM, M. N.; DUFRESNE, A. Short natural-fibre reinforced polyethylene and natural rubber composites: effect of silane coupling agents and fibre loading. Compos Sci Technol, 67(7-8), p. 1627-39, 2007.

ASHBY, M. F.; JOHNSON, K. Materials and design: the art and science of material selection in product design. Amsterdam: Elsevier/Butterworth-Heinemann, 2010.

AVÉROUS, L.; BOQUILLON, N. Biocomposites based on plastificized starch: thermal and mechanical beharviours. Carbohydrate Polymers, vol. 52, p. 111-122, 2004.

AVÉROUS, L.; DIGABEL, F. Properties of biocomposites based on lignocellulosic fillers. Carbohydrate Polymers, vol. 66, p. 480-493, 2006.

BEYLERIAN, G. M.; DENT, A. Ultra materials: how materials innovation is chanching the world. Kingdom: Thame \& Hudson, 2007.

CALLISTER, W. D. Ciência e Engenharia de Materiais: Uma Introdução. São Paulo: LTC, 2002.

CAMPOS, A. de et al. Efeito do tratamento das fibras nas propriedades do biocomposito de amido termoplástico/policaprolactona/sisal. Polímeros, v. 21, p. 217-222, 2011.

CARVALHO, L. F. M. Tratamentos de fibras de carnaúba [copernicia prunífera (miller) H. E. moore] para o desenvolvimento de compósito biodegradável com matriz de polihidroxibutirato. 2011. $105 \mathrm{f}$. Tese (Doutorado) - Universidade Federal do Rio Grande do Norte, Programa de Pós-Graduação em Ciência e Engenharia de Materiais.

FARUK, O. BLEDZKI et al. Biocomposites reinforced with natural fibers: 2000-2010. Progress in Polymer Science, 2012.

$K A B I R, M . M$. et al. Chemical treatments on plantbased natural fibre reinforced polymer composites: An overview. Composites: Part B 43, p. 2883-2892, 2012.

KAZAZIAN, T. Haverá a idade das coisas leves: design e desenvolvimento sustentável. São Paulo: Editora Senac São Paulo, 2005.

MANZINI, E.; VEZZOLI, C. O desenvolvimento de produtos sustentáveis: os requisitos ambientais dos produtos industriais. São Paulo: Edusp, 2002.

MOHANTY, A. K. et al. Natural Fibers, Biopolymers, and Biocomposites: An Introduction. Taylor \& Francis, 2005.

MOHANTY, A. K.; MISRA, M.; HINRICHSEN, G. Biofibres, biodegradable polymers and biocomposites: An overview. Macromol. Mater. Eng. 276/277, p. 1-24, 2000.

OLIVEIRA, M. Novo uso para a glicerina se desenvolve. Disponível na internet por http em:<http://www.biodieselbr.com/noticias/biodiesel/novo-uso-glicerina-desenvolve-407-08.htm> Acesso em 11 maio 2013.

PLACKETT, D. et al. Biodegradable composites based on L-polylactide and jute fibres. Composites Science and Technology, 63 (9), p. 1287-1296, 2003. 
RAMÍREZ, M. G. L. Desenvolvimento de biocompósitos de amido termoplástico reforçados com fibra de coco verde. 2011. $148 \mathrm{f}$. Tese (Doutorado) - Universidade federal do Paraná, Programa de Pós-Graduação em Engenharia Florestal.

ROSA, M. F. et al. Compósitos biodegradáveis reforçados com fibra de coco imaturo. Embrapa Instrumentação Agropecuária, São Carlos, 2009.

SCHLEMMER, D.; de OLIVEIRA, E. R.; ARAÚJO SALES, M. J.Polystyrene/thermoplastic starch blends with different plasticizers. Journal of Thermal Analysis and Calorimetry, v. 87, p. 635-638, 2007.

SATYANARAYANA, K. G. Biodegradable polymer composites based on Brazilian lignocellulosic. Revista Matéria, v. 15, n. 2, p. 088-095, 2010.

SILVA, H. S. P. Desenvolvimento de compósitos poliméricos com fibras de curaua hibridos com fibras de vidro. 2010. 86 f. Dissertação (Mestrado) - Universidade Federal do Rio Grande do Sul , Programa de Pós-Graduação em Engenharia de Minas, Metalúrgica e de Materiais.

VILAPLANA, F. et al. Environmental and resource aspects of sustainable biocomposites. Polymer Degradation and Stability, p. 2147-2161 , 2010. 